\title{
EchoGéo
}

$37 \mid 2016$

Femmes et migrations : celles qui restent

\section{La géographie du collège à l'épreuve des récits}

Des programmes à venir entre éthique professionnelle et tradition disciplinaire

Jean-François Thémines

\section{OpenEdition}

Journals

Electronic version

URL: https://journals.openedition.org/echogeo/14651

DOI: $10.4000 /$ echogeo. 14651

ISSN: 1963-1197

Publisher

Pôle de recherche pour l'organisation et la diffusion de l'information géographique (CNRS UMR 8586)

Electronic reference

Jean-François Thémines, "La géographie du collège à l'épreuve des récits", EchoGéo [Online], 37 | 2016, Online since 07 October 2016, connection on 10 August 2021. URL: http://journals.openedition.org/ echogeo/14651; DOI: https://doi.org/10.4000/echogeo.14651

This text was automatically generated on 10 August 2021

EchoGéo est mis à disposition selon les termes de la licence Creative Commons Attribution - Pas d'Utilisation Commerciale - Pas de Modification 4.0 International (CC BY-NC-ND) 


\title{
La géographie du collège à l'épreuve des récits
}

\author{
Des programmes à venir entre éthique professionnelle et tradition \\ disciplinaire
}

Jean-François Thémines

1 Au printemps 2015, les projets de programmes d'histoire du collège ont donné lieu à de vifs échanges publics dans un contexte marqué par les attentats de janvier. Ces échanges ont fait jouer la fibre nationale sans toutefois concerner les programmes de géographie. Rien a priori n'empêchait que la question de la place, de la fonction et de la nature du récit national dans l'histoire scolaire ne soit aussi adressée, avec les inflexions disciplinaires de rigueur, à la géographie scolaire. Quelle place celle-ci accorde-t-elle au territoire national et à son étude? Quelles images en sont enseignées? Que connaissent les élèves en matière de géographie de la France, en fin de collège aujourd'hui? Mais il n'en a rien été.

2 Parallèlement, on constate un fort intérêt pour des essais et des documentaires qui prennent l'espace comme angle d'analyse de la société française. Qui est Charlie? (Todd, 2015) ; quelques mois auparavant La France périphérique (Guilluy, 2014); un peu plus tôt, de nombreux «voyages en France» (Aubenas, 2014; Bailly, 2011; Dupin, 2010), la France de Raymond Depardon (Depardon, 2010), etc. Même si la plupart de ces auteurs ne sont pas géographes, la géographie est, d'une certaine façon, là. Outre qu'ils questionnent la société française à partir de la dimension spatiale, ces essais ont un caractère de récit; si l'on entend par récit, la représentation d'un ensemble d'événements, sous la forme d'une histoire qui permet au lecteur de les ressaisir en un tout signifiant (Adam, 1984). Dans ces récits, l'espace et les lieux ne sont pas le décor, mais les personnages et les ressorts de l'histoire.

3 Cette actualité éditoriale tranche avec le silence qui accompagne la parution des programmes de géographie. Le silence est d'autant plus notable qu'aux côtés de l'histoire, la géographie scolaire a longtemps ancré le récit de la construction nationale à une description détaillée du territoire. Dès lors, deux façons de concevoir la place du récit géographique en collège, lorsqu'il s'agit d'étudier la société française, sont 
possibles. La première est une conception spontanéiste. L'enseignement de contenus produit naturellement, par sa force propre et ses outils habituels éventuellement rénovés (cartes, photographies de paysage, schémas, globes virtuels, photorécits), un récit unique dans lequel chacun est tenu de s'inscrire. La seconde est une conception critique. Elle consiste à penser qu'un récit géographique, nécessaire parce qu'il rend perceptible la perspective d'un commun, ne va nullement de soi, appelle enquête et discussion, mise en visibilité et travail critique ${ }^{1}$. C'est cette deuxième perspective que le texte cherche à jalonner.

Quels enjeux politiques et pratiques peut-on identifier autour de la fabrique scolaire de récits dans les classes de géographie lorsqu'il est question de la société française?

Nous partirons des programmes de collège de 2008 d'où toute perspective de récit semble absente pour aller vers les récits géographiques actuellement édités, afin d'en dégager quelques enjeux de formation scolaire. À partir de ces enjeux, nous reviendrons alors vers le collège, cette fois-ci avec les programmes de 2016, pour cerner les possibilités qu'ils ouvrent aux professeurs d'histoire-géographie en matière de construction d'un commun.

\section{La présence indécise de la géographie au collège}

Les programmes de géographie du collège ont fortement évolué en 2008. Alors qu'ils ont longtemps obéi à une logique de découpage du monde par continent et par pays, des apparentements avec la géographie scientifique (l'habiter en classe de sixième, les inégalités en classe de cinquième, la géographie de la mondialisation en classe de quatrième) les ont fortement renouvelés. À la faveur de cette réécriture, les contenus de géographie de la France et de l'Europe ont été placés en classe de troisième, où ils closent symboliquement le parcours commun à l'ensemble des publics scolarisés. Pour autant, une discipline scolaire se définit et se légitime d'abord par les finalités qui orientent ses contenus et justifient leur présence scolaire (Prost, 1998). La réception bruyante des projets de programmes d'histoire pour la rentrée 2016 indique que c'est par l'histoire que le couple scolaire histoire-géographie est majoritairement appréhendé. Ce statut de matière seconde de la géographie peut être rapporté, même si cela ne suffit pas à l'expliquer, à l'indécision qui caractérise sa présence scolaire au secondaire (Grataloup, 2015).

7 Depuis la III ${ }^{e}$ République et pendant près d'un siècle, la géographie scolaire est censée avoir contribué de manière spécifique au partage d'un récit national. L'acteur principal de ce récit est la nation française, dont la géographie montre à travers des images de paysages et des cartes, les vertus et la capacité à se forger un destin collectif. C'est ce récit que les générations successives d'élèves ont été conduites à partager, semble-t-il ; même si d'anciens élèves montrent que chacun pouvait à partir des pratiques professorales penser et rêver sa propre inscription dans l'espace français (Ozouf, 2009 ; Sansot, 1985). Les travaux d'histoire de la géographie scolaire ont permis d'établir que l'iconographie des manuels scolaires et universitaires a servi ce type de récit en valorisant le centre politique et historique du pays ainsi que ses limites (Mendibil, 1997). Pourtant, les travaux de didactique de la géographie montrent qu'à partir des années 1970, la discipline, en mal de légitimation, est « marquée par la recherche d'une sorte de rationalité instrumentale ( (Audigier, 1993, p. 38). Elle tend (pas seulement la géographie, mais l'histoire-géographie) à se constituer, au moins dans les prescriptions 
institutionnelles, en instance de transmission d'une "boîte à outils". Les textes officiels livrent la liste des outils (repères, notions, méthodes) à acquérir par les élèves pour développer leur autonomie de pensée. «Cette boîte à outils ne traite du sens de ces savoirs que de façon fort lointaine. C'est à chacun de faire le lien entre les savoirs scolaires et son destin individuel et collectif » (Audigier, 1995, p. 76).

8 S'agissant de la géographie de la France, tout semble se passer dans l'enseignement tel que les programmes le cadrent, comme si la possibilité d'un récit devait résulter, naturellement pour ainsi dire, de l'enchaînement des thèmes mis à l'étude. Les programmes de la classe de troisième de 2008 (Bulletin officiel, numéro spécial n ${ }^{\circ} 6 \mathrm{du}$ 28 août 2008) proposent une succession d'approches distinctes par les échelles, les concepts-clés et les problématiques : les aires urbaines et les espaces ruraux, la région, la population de la France, les espaces productifs, les contrastes territoriaux, la France dans l'Union européenne et le monde. Ils ne mentionnent pas de travail spécifique concernant ce que le mot France désigne ou évoque comme idées, comme problèmes et les éclairages que peut apporter la géographie sur de tels sujets. Si on s'en tient à la lettre des programmes de 2008, la géographie de la France est un moyen comme un autre pour mettre en œuvre des catégories, des démarches et des techniques propres à la géographie scolaire ; un compartiment de la boîte à outils en quelque sorte.

Est-ce que tout récit géographique a disparu des classes parce que les textes officiels sont muets à ce sujet et/ou parce qu'il est inadapté aux enjeux contemporains de construction d'identités individuelles et collectives ainsi que de représentations partagées? Ou bien, doit-on considérer le récit comme une ressource encore opérationnelle pour relever ce type d'enjeux dans les classes? Et dans ce cas, de quels récits géographiques peut-il s'agir aujourd'hui et comment y travailler avec des élèves en collège?

\section{L'actualité française des récits géographiques de pays}

10 Nous n'aurions pas insisté sur ces questions si le silence autour des programmes de géographie n'avaient pas été contemporains du succès d'ouvrages qui proposent des lectures de la société française par l'espace et les lieux. En quoi cet ensemble d'ouvrages éclaire-t-il les enjeux de récit dans les classes de géographie pour l'enseignement de la France?

\section{Ce qui s'impose, dès lors, c'est d'aller y voir ${ }^{2}$}

Dans cette catégorie du récit géographique de pays, la narration est configurée par une enquête représentée comme conduite en des lieux ou à partir de localisations jugées significatives de problématiques sociales. Comme tout récit, elle «ne trouve son sens qu'à accomplir un certain effet sur celui (ou ceux) à qui (auxquels) il est destiné » (Adam, 1984, p. 11). Ici, c'est un lecteur placé tout à la fois face à l'image d'une société qu'il connaît ou pense connaître bien et comme à l'intérieur de cette image - ou s'attendant à devoir s'y retrouver. De ce fait, le récit géographique de pays met en branle des jeux de reconnaissance ou de non reconnaissance de soi et de son environnement (est-ce de mon pays dont on me parle ? Finalement, de quel pays me parle-t-on?). Il est intéressant parce qu'il permet de repérer des manières de 
construire une image de la société française par l'espace, manières fondées sur des conceptions fort disparates.

L'outil de repérage de ces conceptions présenté dans l'illustration 1 est organisé par l'intersection de deux axes d'intelligibilité. Le premier axe se rapporte au statut de l'espace dans l'énonciation narrative (qui accomplit le récit?). Il rend compte de la capacité que l'auteur accorde à son lecteur de contribuer au récit qu'il lui adresse, voire d'y prendre part et de le prolonger. Dans quelle mesure l'auteur lui accorde-t-il, lui reconnaît-il, en particulier à travers le portrait qu'il fait des habitants du pays, cette possibilité de contribution? Le deuxième axe se rapporte au statut de l'espace dans la configuration narrative (quelle mise en intrigue de l'action?). Il rend compte de la conception de l'espace mobilisée par l'auteur pour l'analyse des pratiques des habitants (parmi lesquels le lecteur). Sur cet axe, est examiné le poids relatif des «contraintes spatiales » et de l'inventivité des habitants. Dans quel rapport le récit place-t-il les unes et les autres?

Illustration 1 - Conceptions de l'espace mobilisées dans des récits de pays

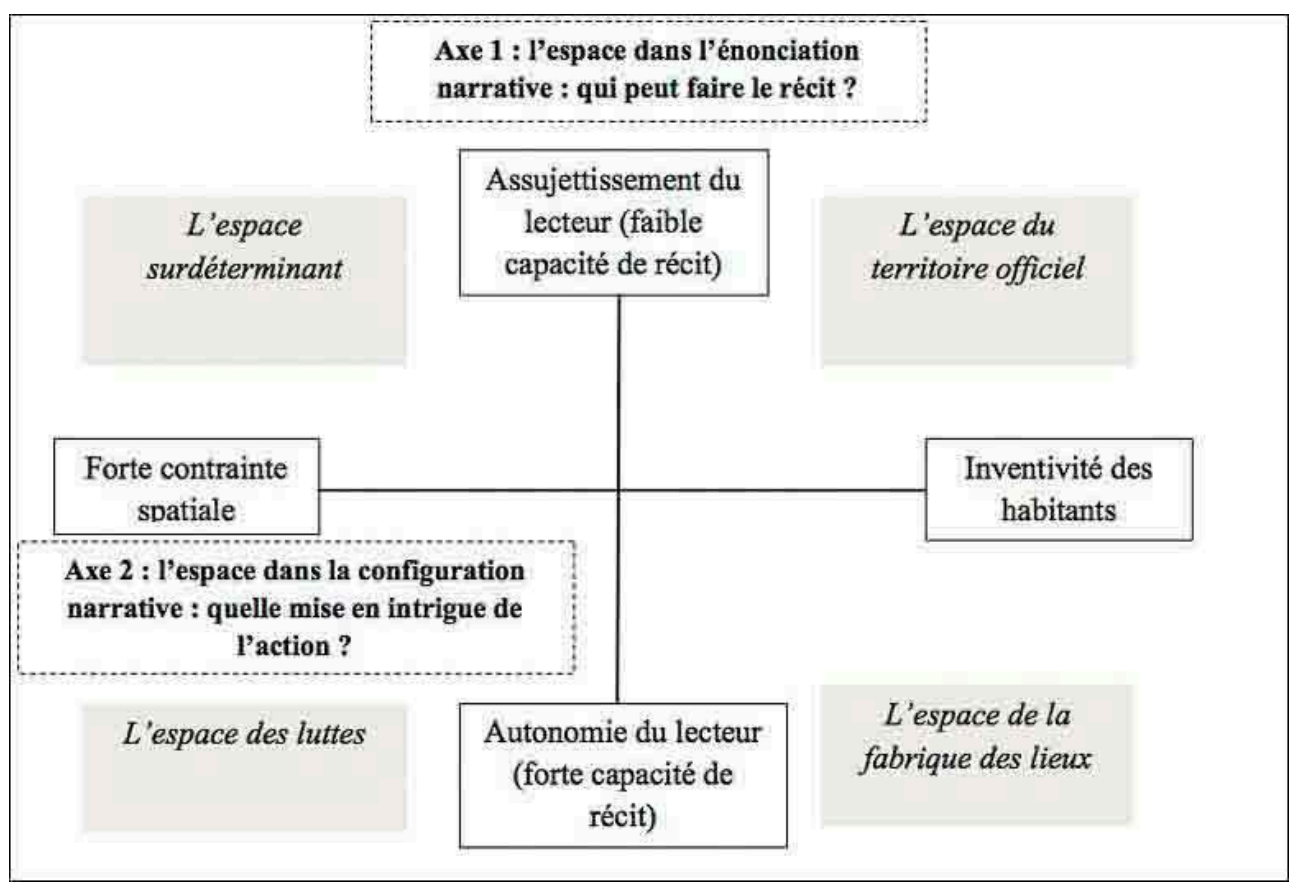

Le premier axe oppose deux images : d'un côté, celle de l'assujettissement du lecteur ou en tout cas sa très faible capacité à infléchir un récit, inféodé qu'il est à des forces d'échelle et d'efficacité supérieures; et de l'autre, celle de l'autonomie du lecteur, sa capacité de production narrative. L'assujettissement correspond à une situation de dépendance totale des individus vis-à-vis de facteurs qui déterminent un grand récit qui les dépasse. Celui qui parle (énonciation narrative) connait cette dépendance ; il est celui qui, sachant, surplombe ceux auxquels il s'adresse. L'autonomie peut être rapportée à la définition d'une des capacités qui sont pour Paul Ricoeur (2006) la première assise de l'humanité : le « pouvoir raconter » dont l'enjeu est pour le sujet la lisibilité et l'intelligibilité des événements. Celui qui parle invite ses interlocuteurs au récit par la place qu'il leur donne, les hésitations qu'il partage, les horizons qu'il ouvre.

Le deuxième axe oppose deux conceptions : celle d'un espace attribut de position qui caractérise une population, et celle d'un espace ressource mobilisé dans la mise en 
œuvre quotidienne ou la défense de modes de vie. D'un côté, dans la configuration narrative, l'espace intervient comme déterminant direct ou indirect des orientations et des choix de vie des personnes. Ce spatialisme se manifeste par le rôle majeur que jouent dans le récit des "territoires-personnages" (des centres, des périphéries auxquels sont conférées des propriétés d'animation). À l'inverse, d'autres récits valorisent les manières de faire avec l'espace d'individus et de collectifs. La configuration narrative met en valeur leurs initiatives ainsi que leurs conflits et confrontations avec des dispositifs institutionnels.

\section{Quatre repères pour des récits géographiques de pays} définies.

L'espace surdéterminant est une conception qui affleure dans les récits d'assujettissement des personnes à des forces supérieures. L'auteur du livre est seul capable d'un récit géographique avec les forces supérieures qu'il met en scène et dont il dévoile le jeu. Le livre d'Emmanuel Todd, Qui est Charlie? Sociologie d'une crise religieuse, est l'expression pure de ce spatialisme. Ce récit d'un affrontement entre deux structures anthropologiques pour le contrôle de la société française s'ancre à une conception essentialiste des territoires : à chaque famille un territoire d'ancrage qui lui transmet ses valeurs immémoriales (Todd, 2015, p. 141-143). Ces structures anthropologiques ancrées gouvernent les individus et par conséquent les lecteurs: tous pareils, des masses manipulées par un inconscient anthropogéographique.

L'espace des luttes correspond à une conception qui soutient l'autonomie des acteurs, entre autres de ceux qui bien que faiblement dotés, s'organisent et écrivent, par l'action en quelque sorte, un récit propre. Comme dans le type précédent, une certaine stabilité de contexte géographique constitue le fond de l'histoire sur lequel cependant, à la différence d'un espace surdéterminant, des acteurs produisent une part de la narration que l'auteur transcrit (c'est comme cela qu'il le présente). La France périphérique. Comment on a sacrifié les classes populaires, l'essai de Christophe Guilluy, relève de ce type. La liste des traumatismes et des mouvements sociaux de la France périphérique des invisibles et des oubliés fait de tel bourg, présent par son seul nom et l'effectif correspondant d'emplois menacés ou perdus, un actant du récit.

L'espace du territoire officiel est une conception qui subordonne les individus et les groupes à des logiques supérieures où l'espace est cependant pensé comme un potentiel. Dans ce cadre, c'est l'exploitation de l'espace, sa mise en forme qui soutient un récit où dominent un ou quelques grands acteurs que les autres suivent. Il n'y a pas d'ouvrage marquant pour incarner ce type de récit proche de l'État. Mais on peut en trouver trace dans Hexagone à géométrie variable, l'article dans lequel Armand Frémont rend compte pour Télérama du travail de Raymond Depardon. Gêné par l'absence de lignes de force et de pôles majeurs, il propose une sorte de contre-récit quasi-officiel partant de l'île-de-France et de Paris pour atteindre la Corse et l'Outre-mer et finalement retrouver une «France de Depardon solidement construite dans les profondeurs de la province » (Frémont, 2010, p. 65).

19 L'espace de la fabrique des lieux correspond à une conception où la ressource spatiale est activée par de "petits » acteurs qui tissent des rapports avec et entre les lieux. Ce type de récit peut être illustré par l'essai de Jean-Christophe Bailly Le dépaysement. Voyages en 
France. À l'issue d'un récit qu'ouvrent de nombreuses rencontres et perspectives, l'auteur fait partager son hésitation face « à l'écartement le plus net et le plus ouvert de ces différences » (Bailly, 2010, p. 338) : «Il me reste un cageot d'endives au coin d'une rue à Roubaix et un Martini dry bu dans un bar d'hôtel à Cannes et l'impossibilité de les relier l'un à l'autre ailleurs que dans ma mémoire. Une sagesse (ou une résignation) me souffle que c'est bien assez, mais il me semble alors que le compte n'y est pas » (ibid., p. 340). D'autres voyages en France (Aubenas, Depardon, Dupin) soutiennent cette conception d'un espace social fragilisé dans ses écarts, mais qui d'un lieu habité à un autre, de récit réalisé en récit latent, n'est pas totalement perdu de vue.

Nous obtenons ainsi l'esquisse d'un tableau de la pluralité des récits géographiques publiés ces dernières années. L'espace surdéterminant signe la disparition du pays en révélant de vastes territoires cachés sous le pavage des États. L'espace des luttes ausculte les fractures vives entre France(s) qui dérivent par blocs. L'espace du territoire officiel est franchement en second plan pour soutenir l'idée d'action unificatrice de l'État dans une France d'individualités multiples et de sentiments contradictoires (Frémont, 2010, p. 66). Enfin, l'espace de la fabrique des lieux décrit la recherche de signaux, de réminiscences et tisse de multiples récits singuliers.

\section{De la pluralité des récits aux enjeux de l'étude géographique de la société française au collège}

21 Cette pluralité de récits géographiques de pays invite à revenir vers la géographie scolaire afin de cerner certains enjeux de l'étude géographique de la société française, que le silence autour des projets de programmes n'a pas aidé à circonscrire.

C'est tout d'abord cette vitalité éditoriale, comprise comme un pan de la réalité sociale, qui semble ne pas avoir été connectée aux débats sur les programmes du collège. Les récits que nous avons évoqués, par leur nombre, leur accumulation, ont installé dans l'horizon d'attente de lecteurs, un genre ou en tout cas un type d'ouvrage (précisément celui que nous appelons le récit géographique de pays). À l'intention persuasive de ces récits, il faut coupler le croire interprétatif du lecteur, lui-même a priori confiant ou méfiant, mais acceptant qu'il s'agit bien d'un récit du genre ou du type que nous avons formalisé (Adam, 1984). Chaque nouvelle occurrence de récit alimente une réception, suscite des discussions à la faveur desquelles se confrontent des visions de la société française. Le récit géographique de pays est ainsi devenu une réalité sociale, bien audelà des arènes universitaires.

Avant même la pluralité des récits, on peut être frappé par le caractère divers et fragmentaire des contenus qu'ils articulent. Cette diversité et cette fragmentation montre que tout de la France n'est pas déjà dit, ni connu, d'un point de vue géographique et qu'il y a donc matière à enquête. On sait même peu de choses des rapports des habitants à la France par les lieux ${ }^{3}$, en particulier de leurs habitants adolescents. Par ailleurs, les essais et documentaires que nous avons évoqués ne sont sans doute qu'une variante du récit géographique de pays. Ce type de récit traverse diverses sphères: celles de l'aménagement et du marketing territorial bien sûr, celle aussi de la presse d'opinion, mais encore la blogosphère et les genres musicaux populaires plus familiers des adolescents. Rendre les classes de géographie étanches à ces textes, c'est prendre le risque de penser qu'il n'existe pas d'enjeu de connaissance des rapports de la société française avec l'espace et avec les lieux. 
24 Après l'enjeu de prise en compte d'une réalité sociale et l'enjeu de connaissance géographique, un troisième enjeu peut être identifié, cette fois-ci autour de la question de la capacité de production de récit. Nous nous rapprochons là du domaine des finalités de la géographie scolaire. Ainsi qu'en témoigne la réception des ouvrages que nous avons mentionnés, toute production de récit est imbriquée dans des situations et des contextes multiples, qu'ils soient politiques, médiatiques, artistiques ou littéraires. L'effet recherché vise des lecteurs agissant dans ou réagissant à ces situations et ces contextes. Une partie des Voyages en France publiés dans les années 2010-2012 répond d'ailleurs au « Débat sur l'identité nationale » organisé par le ministre de l'Immigration et de l'Identité nationale du gouvernement Fillon, Eric Besson, entre octobre 2009 et février 2010. La réalité sociale que constituent les récits géographiques de pays est aussi une réalité en action(s): elle participe à la configuration conflictuelle de l'espace français. Mais, comme nous avons pensé l'avoir montré, les auteurs des récits ne placent pas également leurs lecteurs en position d'être à leur tour des auteurs de récits (par conséquent des acteurs). Certains auteurs les enferment dans l'impuissance à comprendre et agir ; d'autres leur reconnaissent des capacités d'action (de récit) et ce faisant soutiennent ces capacités. L'enjeu de l'assujettissement versus l'émancipation nous parait fondamental et il peut être exactement transposé en termes de finalités de formation géographique des élèves, s'agissant de leur capacité d'analyse et de production de récit.

Un quatrième et dernier enjeu recoupe les trois précédents, tout en les replaçant cette fois-ci sur le terrain de la professionnalité des enseignants en géographie. Par professionnalité, nous entendons un «système de normes et de valeurs en acte [...] liée à l'histoire du métier, à l'état des attentes socio-politiques, aux environnements de travail et aux modifications du prescrit " (Lantheaume et Simonian, 2012). Entendons qu'elle se définit du point de vue des professionnels, de celles et ceux qui enseignent, qu'elle n'évolue pas sur le modèle de la «bascule » privilégié par les décideurs, mais que les pratiques procèdent de traductions des prescriptions, généralement éclairées par des normes préalablement établies. S'agissant de l'absence apparente de connexion entre les récits géographiques de pays et l'enseignement de la géographie de la France, la question posée est celle de la ressource de professionnalité qui se trouve ainsi tue ou inhibée. Deux options sont possibles. Premièrement, l'enjeu idéologique du récit national en histoire a focalisé les prises de position de professionnels au détriment d'une mise en visibilité de réflexions collectives conduites par ailleurs sur l'étude géographique de la société française. Dans ce cas, il y a bien des normes et des valeurs en place, éventuellement divergentes, qui conduisent à travailler dans les classes sur et avec les points de vue existants à propos du fonctionnement et des évolutions de la société française. Deuxièmement, l'étude géographique de cette dernière est essentiellement orientée par le passage en revue d'objets disparates indiqués par les programmes de 2008. Dans ce cas, elle n'est pas un thème porteur de débats et d'acquis de professionnalité pour les professeurs d'histoire-géographie.

Quelle que soit la réponse, nous cernons ainsi ce que nous appelons un enjeu de ressource de professionnalité. À savoir : comment l'étude géographique de la société française contribue-t-elle - ou non - à une définition par les professeurs d'histoiregéographie, de leur éthique du métier ? Cet enjeu fournit l'angle à partir duquel nous allons maintenant examiner les programmes de géographie de la rentrée 2016. 


\section{Géo-éthique professionnelle et tradition disciplinaire}

27 Comment l'enseignement de la géographie de la France est-il prescrit par les programmes de géographie de 2016 ? Est-ce que cette prescription, enregistrant en quelque sorte sans le dire l'existence de pratiques souhaitées, officialise et encourage de nouvelles perspectives professionnelles? Dans quelle mesure des enjeux du type de ceux identifiés à partir des récits géographiques de pays sont-ils pris en compte et peuvent-ils être relevés dans l'enseignement de la France?

\section{Une logique combinatoire limitée en géographie pour les programmes de 2016}

Répondre à ces questions suppose de cerner la nouvelle notion de programme qui structure les enseignements prévus au collège à partir de la rentrée scolaire 2016. Les programmes sont désormais organisés en trois "volets " ${ }^{4}$ (voir illustration 2). Cette architecture traduit l'intention formulée par le géographe et président du Conseil National des Programmes Michel Lussault de faire du socle « une plateforme générative ${ }^{5}$.

Illustration 2 - Le Programme d'enseignement du cycle des approfondissements : organisation de son écriture

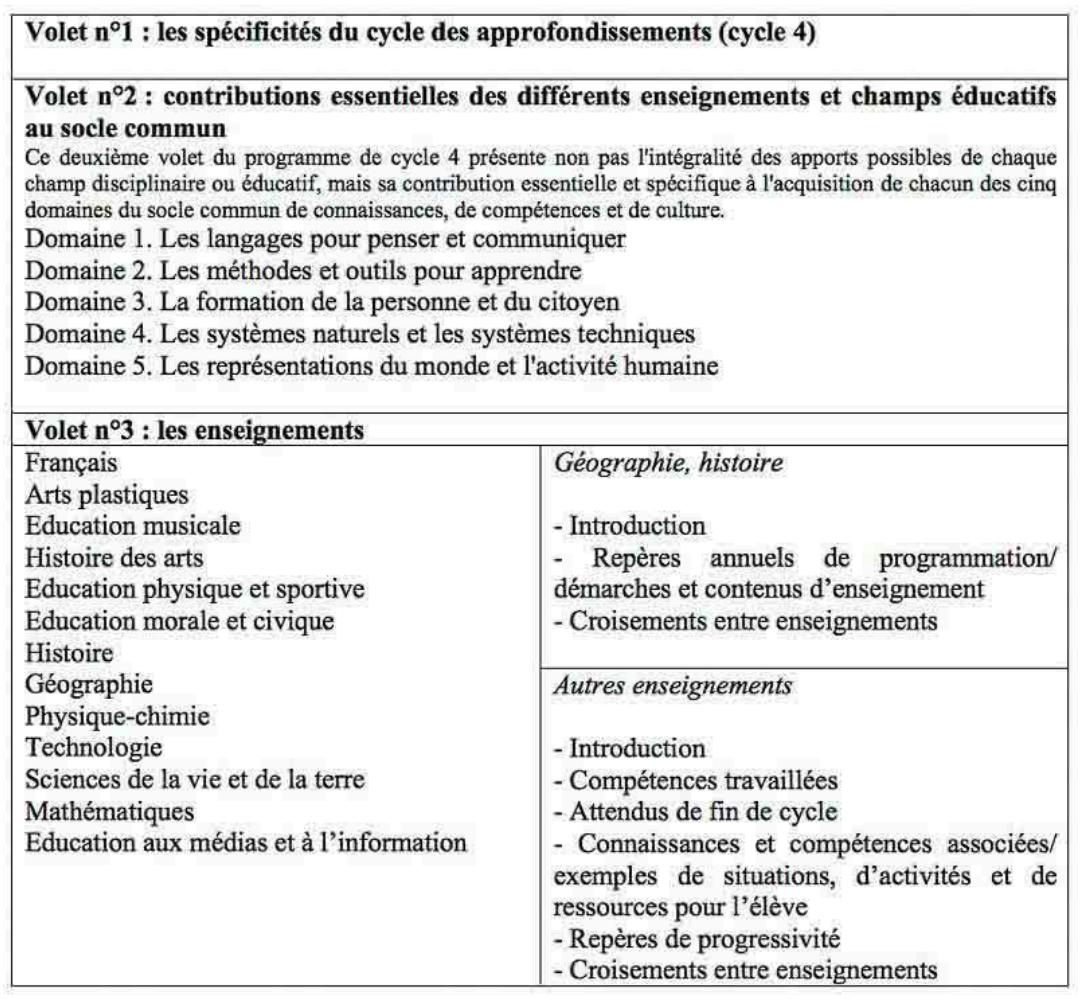

Source : Bulletin Officiel spécial du 26 novembre 2015.

29 À la différence du précédent socle commun (2006) qui conservait de forts repères disciplinaires répartis entre "piliers ", les cinq domaines de ce nouveau socle sont à combiner pour l'ensemble des enseignements. Le domaine $n^{\circ} 5$, intitulé Les représentations $d u$ monde et l'activité humaine, apparaît comme un pivot pour la 
géographie. Il demande de former à la pensée critique et à la controverse ainsi qu'à la compréhension de systèmes complexes produits par les sociétés. Il demande aussi de faire apprendre des repères, de permettre de se situer dans le monde social comme acteur et de se projeter dans le futur. Le domaine $\mathrm{n}^{\circ} 4$, appelé Les systèmes naturels et les systèmes techniques sollicite la géographie sur le thème des risques et sur « la démarche de questionnement en donnant à imaginer des stratégies de sélection des informations reçues en classe, en les croisant avec ses représentations pour expliquer [...] l'organisation d'un territoire ». Les apports du domaine $\mathrm{n}^{\circ} 3$, La formation de la personne et $d u$ citoyen se condensent en un triptyque: questions vives, valeurs partagées et appropriation des questions de justice. Intitulé Les méthodes et outils pour apprendre, le domaine $n^{\circ} 2$ est au service des autres domaines. On peut y souligner la compétence d'évaluation et de traitement de l'information, le caractère coopératif et collaboratif du travail scolaire, enfin la réalisation de projets. Le domaine $n^{\circ} 1$, Les langages pour penser et communiquer, outre qu'il rappelle les spécificités des langages utilisés en géographie comme en histoire, insiste sur deux de leurs fonctions: permettre de passer du spontané au réfléchi, permettre la communication et l'échange de vues.

Pris seul, ce volet ouvre de nombreuses possibilités et autoriserait que l'on s'appuie sur le potentiel des récits géographiques de pays. On peut imaginer deux pistes, parmi bien d'autres, qui permettraient de lier des apprentissages disciplinaires contribuant aux cinq domaines composant le nouveau socle (langages, méthodes, formation de la personne et du citoyen, fonctionnement des systèmes, représentations) :

- la construction par la classe d'un récit géographique de pays. La question de sa possibilité est alors authentiquement posée : peut-on, par des lieux partagés ou qui fonctionneraient en résonance, tramer les récits de chacun? Quelle place fait-on aux lieux des autres et comment? Comment les savoirs géographiques scientifiques sont-ils mis en lien et contribuent-ils à structurer et fonder les récits?

- la présentation cartographique d'une géographie des inégalités et/ou d'une géographie des initiatives citoyennes de justice sociale. De nombreuses questions de méthodologie sont alors à aborder, de la production et de la vérification des bases de données à cartographier à la maîtrise du raisonnement avec des cartes et aux choix des cartes finalement présentées et débattues $^{6}$, mais aussi des questions de référentiel éthique, en l'occurrence les manières de définir la justice et d'en déduire des critères qui fondent la réalisation de ces cartes.

31 L'articulation entre le volet suggérant les contributions des différents enseignements au socle commun et le volet enseignements pose néanmoins des questions d'opérationnalisation pour la géographie. Le mode d'écriture des contenus de cet enseignement est en effet, de même que celui de l'histoire et à la suite de celui-ci, différent du mode d'écriture général qui est celui de tous les autres enseignements. La logique générale consiste à présenter les enseignements comme contribuant aux apprentissages scolaires attendus. Cette contribution est déclinée en connaissances et compétences associées. Elle est éclairée par des listes et catégories de situations d'activités et de ressources pour les élèves. Des repères de progressivité et des attendus de fin de cycle précisent les performances à atteindre à la fin du collège. En revanche, pour la géographie comme pour l'histoire et l'histoire des arts, la notion de programme annuel est maintenue au détriment de la perspective d'une progressivité sur le cycle. Structurée en thèmes, la présentation des contenus ne comporte pas de situations d'apprentissage. L'écriture de " démarches et contenus d'enseignement » oscille entre condensé de cours et évocation elliptique d'un récit de classe centré sur le professeur. 
Dans cette portion du texte officiel comme nulle part ailleurs, le professeur «montre aux élèves ", « insiste sur... ", " présente aux élèves ", « livre aux élèves les bases des notions".

Faute de situations d'apprentissage dûment nommées, le travail opéré avec les élèves est décrit comme visant une sensibilisation (11 occurrences du mot « sensibiliser » sur les 14 que comprend l'ensemble des programmes concernent la géographie ${ }^{7}$ ). Savoir comment ce mode d'écriture qui fait décrocher la géographie (et l'histoire) du reste des enseignements, s'est imposé, dans le cadre de quel rapport de forces et à quel moment, n'est pas notre question. Nous observons seulement le maintien propre à ces deux disciplines d'une distribution de contenus d'ailleurs inchangée pour chacune des quatre années du collège ${ }^{8}$. Cette rigidité représente une forte contrainte quand il est en même temps demandé aux professeurs de combiner différents cadres (les enseignements, les domaines du socle, les parcours transversaux, les enseignements pluridisciplinaires). C'est précisément lorsque le volet enseignement s'ouvre pour terminer sur des croisements, que l'on perçoit le mieux en géographie, des possibilités de diversification de situations et par conséquent de contenus.

\section{La valeur du récit géographique : une situation d'apprentissage sur la France en géographie}

33 Ce que les élèves apprennent en classe de géographie dépend entre autres des situations que les professeurs conçoivent. En ne nommant pas de situations d'apprentissage en géographie (ou en histoire), les textes officiels perpétuent une écriture désuète de l'enseignement et des effets qu'il vise (des apprentissages clairement désignés). Cela ne veut pas dire qu'il n'existe pas un « répertoire » d'actes professionnels. Ce n'est certainement pas à des textes institutionnels de le faire, même si on peut se demander quel est l'intérêt pour un ministère de publier un texte qui mime faussement des situations professionnelles. Même si les réseaux informels d'entraide et de mutualisation entre professeurs pourvoient partiellement à l'identification de telles situations, c'est cette direction de travail, celle d'un répertoire d'actes professionnels, que nous souhaitons baliser à partir du cas de l'enseignement de la géographie de la France. Tandis que celui-ci revêt selon nous un enjeu de ressource de professionnalité, les programmes de la classe de troisième de 2016, corsetés dans une écriture qui ne définit pas les situations du point de vue des élèves reconduisent pratiquement ceux de $2008^{\circ}$. Aussi, concrétiserons-nous cet enjeu à partir d'une situation d'apprentissage qui a été expérimentée en 2012 en s'affranchissant d'une lecture littérale de ces programmes. Sa présentation permettra dans un second temps, par inférence à partir d'éléments amenés par les textes officiels de 2016 en marge des enseignements, de cerner les contours formels d'un répertoire de ce type.

La situation que nous décrivons utilise quatre heures de cours pour un travail de réflexion de la part des élèves sur ce qu'est la France. Elle se situe en début d'année, mais intervient après que l'année précédente, les élèves aient déjà eu cours sur la France: c'est la première année de mise en place de ce nouveau programme de troisième. La tâche consiste à représenter la France sur un panneau grand format à l'aide de documents que les élèves sélectionnent librement. Il leur est précisé que l'organisation du panneau est entièrement de leur responsabilité. Le contrat stipule aussi que le choix de chaque document doit être justifié, argumenté, et que deux textes 
au moins doivent être choisis. Chaque élève dispose de la même surface de panneau et de quatre heures maximum pour parvenir à un résultat.

Les élèves sont confrontés à un grand nombre de «documents »: photographies, représentations cartographiques, revues grand public consacrées à des régions (Terra Corsa, Destination Provence, Bretagne Magazine, etc.), et textes d'auteurs variés. Le choix documentaire est proposé dans les meilleures conditions possibles. Les documents sont répartis par types selon une disposition en ilot, pour que les élèves puissent librement tourner autour. Le corpus est composé de 48 photographies extraites de manuels scolaires ou provenant de sites internet à vocation touristique, de photographies de Raymond Depardon - l'ouvrage La France de Raymond Depardon étant posé sur une table, ainsi qu'un numéro Hors-Série de Télérama, au titre identique. L'ouvrage Paysans du même photographe complétait cette collection d'images d'auteur. Le corpus comprend aussi des représentations cartographiques, toutes représentatives de l'iconographie scolaire, auxquelles s'ajoutent deux cartes routières IGN à l'échelle nationale et un exemplaire d'emporte-pièce en "forme de France». Sept textes complètent l'ensemble, extraits d'ouvrages ou d'articles écrits par des géographes (Armand Frémont, Michel Lussault), un sociologue (Pierre Sansot), des écrivains (JeanChristophe Bailly, Georges Perec, Stéphane Audéguy) et des journalistes (Françoise Fressoz et Thomas Wieder). Enfin, dix revues présentant des régions françaises, sont aussi disposées sur la table documentaire.

Ce dispositif simple aboutit à des productions riches dont nous livrons ici une partie de l'analyse qu'en fait leur professeur. Aussi nous reprenons d'Emmanuel Dugué (2014) deux portraits d'élèves.

Wanda et la France du chômage

Wanda fait reposer son récit sur le thème du chômage. Il est entièrement organisé par une "démonstration » : le chômage est le problème numéro un et les classes moyennes ont peur de la mondialisation. Cette volonté de démontrer est soulignée par l'usage de symboles algébriques $(+,=)$ et de flèches. Elle relie des extraits du Nouvel espace français de Daniel Noin avec un article de presse consacré aux classes moyennes. Elle s'identifie ainsi à une France qui fait peur et qui a peur ; une France qui inquiète et attire à la fois. Wanda sait en effet qu'elle devra bientôt s'insérer dans ce territoire lu à partir du chômage [elle veut obtenir un BEP et travailler aussitôt après]. Ainsi, pour elle, s'identifier à la France, c'est peut-être à la fois, la craindre parce qu'on va bientôt s'y « plonger » et qu'on aimerait la voir autrement, et la revendiquer parce qu'elle s'y sent attachée (Dugué, 2014).

Bastien et la France Into The Wild

Bastien convie à une incursion dans la beauté de la France. Bastien a soigneusement sélectionné deux textes : un extrait d'interview de Michel Lussault et un court passage de La France sensible de Pierre Sansot. Il a du mal à rendre raison de ce choix; mais l'habitude scolaire est de justifier a minima. Les deux grandes photographies qui forment l'assise de son récit ont été très minutieusement choisies dans La France de Raymond Depardon. Il est le seul à avoir adopté ce type de cliché : il a fait le choix de s'exprimer sur ce qu'il valorise avant tout. Ainsi sa France serait une France des grands espaces, une France américaine, Into the Wild en quelque sorte. La France est faite de lui, de ses centres d'intérêt, de son rapport à l'espace. Bastien aime pêcher, chasser mais surtout être dans la "nature ». Il souhaite en faire un élément de sa vie professionnelle future, qu'il imagine comme technicien de la faune sauvage ou travaillant pour l'office National des Forêts (Dugué, 2014).

Où l'on voit la possibilité pour des adolescents de lier leur expérience, tout particulièrement lorsqu'il s'agit de se projeter, aux connaissances de personnes qui font 
profession de produire des savoirs géographiques. Où l'on comprend que les discours ainsi produits (celui de Wanda, celui de Bastien, ceux des autres), permettent d'engager une discussion et des développements sous plusieurs angles: la sélection et l'organisation des contenus; la compréhension et l'usage des arguments scientifiques repris aux auteurs ; la capacité de l'auteur (l'élève) à ouvrir son récit à d'autres propos que les siens; sa capacité de persuasion ; la dimension éthique du texte (mise en rapport de ce qui est avec ce qui devrait être selon une conception de la justice à expliciter et à discuter). Où l'on voit enfin que les élèves produisent des récits singuliers qu'ils rapportent à des textes de spécialistes.

\section{Pour une perspective de géo-éthique professionnelle}

38 La pratique que nous décrivons, par laquelle de jeunes adolescents vont, quelquefois, dévoiler des projets personnels et professionnels, est-elle admissible dans une classe de géographie de collège ? Si l'on s'en tient aux programmes de 2008 qui étaient alors en vigueur, elle ne correspond à aucun des contenus d'enseignement mentionnés. Elle est par contre en adéquation avec une des ouvertures permises par les programmes de 2016. Et surtout, mise en rapport avec cette ouverture, elle permet de formaliser un de ces problèmes éthiques caractéristiques des métiers "vivants", c'est-à-dire dans lesquels on débat de la nature des actes professionnels, du souhaitable et des moyens pratiques de l'atteindre.

Le texte de 2016 indique pour la géographie en classe de troisième que « les approches de prospective territoriale sont particulièrement intéressantes pour sensibiliser les élèves à la portée de l'aménagement et aux débats qu'il suscite ». Par ailleurs, parmi les enseignements interdisciplinaires, concernant le thème Dynamiques territoriales de la France contemporaine, il propose une contribution au parcours avenir sur la thématique du Monde économique et professionnel. Le parcours avenir est le dispositif d'information, d'orientation et de découverte du monde économique et professionnel à proposer à chaque élève de collège. Il est ainsi suggéré de mettre en rapport chez les élèves la capacité à se projeter dans un futur professionnel et la capacité à appréhender et analyser les enjeux de la prospective territoriale. Ce qui, sur le papier, peut paraître une vue de l'esprit, prend un relief particulier à la lumière de la séquence précédemment décrite. L'enseignement de la géographie doit être considéré comme situé, en particulier par rapport aux espaces de pratiques et de socialisations des adolescents scolarisés. La situation proposée a permis à Wanda et Bastien d'exprimer géographiquement des ambitions que d'aucuns jugeront limitées, mais ils l'ont fait en fonction de leurs pratiques et de visions de l'avenir socialement déterminées.

Enseigner la géographie, est-ce alors 1) ignorer le problème ainsi posé ou éviter de (se) le poser en ne construisant pas de situation telle que celle décrite précédemment ou 2) affronter le problème, ce que le texte de 2016 incite à faire, en sachant qu'il va placer le professeur devant un dilemme dont les termes précis sont fonction de son contexte de travail. Par exemple : comment obtenir un effet qui sera de l'ordre de l'engagement ${ }^{10}$ chez les élèves (engagement à l'école) plutôt que de l'ordre du fatalisme, alors que l'analyse géographique de la situation territoriale locale et régionale dévoile, le cas échéant, des phénomènes de déclin, d'anomie, de domination (c'est le cas du contexte de travail du professeur que nous avons cité) ? Comment solliciter - encore et toujours la géographie pour argumenter dans un cours transversal ou interdisciplinaire, en 
faveur d'études longues, à rebours des représentations parentales et adolescentes ? En quoi l'enseignement de la géographie peut-il contribuer à se positionner, comme professeur principal assurant des fonctions d'orientation, face à un modèle social local - absolument légitime et contre lequel se prononce l'institution scolaire aux divers échelons dont le local - de faible mobilité résidentielle et sociale?

Mais à la différence de ce cas de figure où une question d'éthique peut être cernée à partir des rapprochements suggérés par le texte officiel, il existe un potentiel de questions dont le repérage est plus difficile du fait de la faible articulation relative des domaines du socle avec le programme de géographie (et d'histoire). Nous ne donnerons qu'une illustration de ce potentiel. Le socle commun insiste, en particulier pour le domaine $\mathrm{n}^{\circ} 3$, sur le mode de rapport avec autrui à construire, soulignant par exemple l'importance de travailler à "l'ouverture aux autres" ou de développer le "souci d'autrui dans les usages du langage ». Pour autant, il est difficile de trouver un écho explicite à ces finalités dans le programme de géographie. Ce n'est pas le cas pour les enseignements de français, dont l'écriture obéit pleinement à la logique combinatoire $\mathrm{du}$ socle. Les quatre entrées du volet de culture littéraire et artistique ainsi libellées : Se chercher, se construire; Vivre en société, participer à la société; Regarder le monde, inventer des mondes; Agir sur le monde, permettent de formuler des "enjeux littéraires et de formation personnelle » autour du rapport " Avec autrui : familles, amis, réseaux », des " confrontations de valeurs ", et même, s'agissant de « La ville, lieu de tous les possibles ?» des «ambivalences de représentations du milieu urbain». Alors, pourquoi ne pas ouvrir, franchement, de semblables questions, à propos par exemple du vocabulaire disciplinaire de la géographie. Est-ce que ce vocabulaire ne comporte pas ou ne renforce pas des formes de rapport à autrui discutables en classe au regard des finalités générales portées par le socle ? Là aussi, reportons-nous à des situations concrètes de classe. Comment, en tant que professeur y exerçant mon métier, vais-je appréhender avec une classe de troisième, l'étude d'espaces désignés et (dis)qualifiés par les mots de périphéries, de marges (urbaines, rurales) ou par des adjectifs qui les connotent négativement? Quel travail est à faire avec les élèves sur les mots de la géographie scolaire et les normes qu'ils portent, et comment peut-on s'y prendre avec les catégories spatiales ainsi que les espaces de vie et les espaces imaginés qui sont les leurs ? Là encore, pas de réponse simple, générique, mais la certitude d'une dimension éthique spécifique qui rehausse le métier, à la condition qu'elle soit reconnue comme lui appartenant.

Si on voulait étiqueter ce travail à peine esquissé de formalisation de dilemmes tels que ceux-ci ou d'autres déjà rencontrés et formulés localement par de nombreux professeurs, alors on pourrait proposer l'expression de géo-éthique professionnelle. Il ne s'agit pas de l'approche géoéthique proposée par Arnaud Brennetot pour analyser les conceptions de la justice spatiale qui structurent le débat public (Brennetot, 2010), mais d'une démarche - développée ou à développer en formation continue éventuellement dans une logique d'intervention avec des professeurs - d'identification des cas de figure où l'enseignement de la géographie est mis à l'épreuve des valeurs et des normes qui le fondent ou devraient le fonder. Le répertoire de ces dilemmes et la casuistique qui devrait en découler restent à établir ou à rendre visible. Pourtant, la rigidification du texte officiel pour les programmes de géographie (et d'histoire) n'encourage guère la profession à s'y atteler. 


\section{Conclusion}

En se focalisant sur le récit national, les débats de 2015 sur les programmes d'histoire ont occulté les enjeux politiques du récit en classe de géographie. Or, pour cette discipline, ces enjeux se déploient de façon spécifique sur le terrain des rapports sociaux dans l'espace.

Généralement, la fabrique scolaire des inégalités sociales est appréhendée à partir de l'insuffisante explicitation par l'école de ses attentes propres; ce qui favorise les familles et les élèves connivents avec elle (Bonnery, 2015). Quand il s'agit de la géographie, se mêlent d'autres ressorts d'inégalités que les récits géographiques de pays aident à cerner et à travailler. Par exemple, l'enjeu de l'inégale capacité à produire un récit recevable, valable et peut-être même acceptable ${ }^{11}$ dans une classe de géographie. Ne pas prendre en compte cet obstacle socialement construit, c'est laisser les élèves aux prises avec des récits édifiants ou sidérants, faisant obstacle à la perspective d'une socialisation politique. Ou encore l'enjeu d'une inégale capacité des élèves à envisager le futur dans un cadre territorial favorable à leur émancipation.

Parus en silence, les programmes de géographie de la rentrée 2016 ouvrent quelques opportunités. Mais leur rigidité de structure, commune avec l'écriture des programmes d'histoire, risque d'en limiter la portée. L'enseignement de la géographie reste au milieu du gué entre, d'un côté, le chantier de la lutte contre les inégalités sociales dans lequel s'inscrit la Loi pour la refondation de l'école (2013), et de l'autre, le risque de reproduction de pratiques inégalitaires (non voulues à l'échelon individuel) par les territoires qu'elles valorisent ou disqualifient, les discours qu'elles autorisent ou censurent et les mises en partage qu'elles organisent ou écartent. C'est là pourtant que se situent les enjeux d'un chantier de professionnalisation que nous avons qualifié ici de géo-éthique, par lequel le métier gagnerait encore en valeur aux yeux de celles et ceux qui le pratiquent.

\section{BIBLIOGRAPHY}

Adam J.-M., 1984. Le récit. Paris, PUF.

Aubenas F., 2014. En France. Paris, Éditions de l'Olivier.

Audigier F., 1993. Les représentations que les élèves ont de l'histoire et la géographie. À la recherche des modèles disciplinaires entre leur définition par l'institution et leur appropriation par les élèves. Thèse, Université de Paris VII.

Audigier F., 1995. Histoire et géographie : des savoirs scolaires en question entre les définitions officielles et les constructions des élèves. Spirale. Revue de recherches en éducation, $\mathrm{n}^{\circ}$ 15, p. 61-89.

Bailly J.-C., 2011. Le Dépaysement. Voyages en France. Paris, Éditions du Seuil.

Bertho R., D’Andréa N., Bailleul H., Guittet C., Chauvet G., Greffier L., Tozzi P., Bectarte H. et Gagnebien A., 2014. Mon paysage quotidien, une pratique ordinaire? Rapport pour le Ministère de 
l'Écologie, du Développement Durable et de l'Énergie, 159 p. URL : http://www.developpementdurable.gouv.fr/Mon-paysage-au-quotidien-une.html

Bonnery S. (dir.), 2015. Supports pédagogiques et inégalités scolaires. Paris, La Dispute.

Brennetot A., 2010. Pour une géoéthique. Éléments d'analyse des conceptions de la justice spatiale. L'Espace géographique, $\mathrm{n}^{\circ}$ 1, p. 75-88.

Depardon R., 2010. La France de Raymond Depardon. Paris, Éditions du Seuil/Bibliothèque Nationale de France.

Dugué E., 2014. De la France faire lieu : travailler sur les rapports d'identification à la France. In Thémines J.-F. (dir.), La France en classe de géographie. Propositions d'élèves et de professeurs. Paris, L'Harmattan, p. 143-165.

Dupin E., 2011. Voyages en France. La fatigue de la modernité. Paris, Éditions du Seuil.

Frémont A., 2010. Hexagone à géométrie variable. Télérama horizons, La France de Raymond Depardon, p. 62-66.

Grataloup C., 2015. La géographie scolaire sans boussole. Le Débat, n 175, p. 69-78.

Guilluy C., 2014. La France périphérique. Comment on a sacrifié les classes populaires. Paris, Flammarion.

Joliveau T., 2015. Où est Charlie ? Ce que montrent réellement les cartes d'Emmanuel Todd. http:// www.slate.fr/story/101827/ou-est-charlie-cartes-emmanuel-todd

Lantheaume F., Simonian S., 2012. La transformation de la professionnalité des enseignants : quel rôle du prescrit? Les Sciences de l'éducation - Pour l'Ère nouvelle, Vol. 45, p. 17-38.

Mendibil D., 1997. Textes et images de l'iconographie de la France (de 1840 à 1990). Essai d'iconologie géographique. Thèse de doctorat, Université de Paris I.

Ozouf M., 2009. Composition française. Retour sur une enfance bretonne. Paris, Gallimard, nrf.

Prost A., 1998. Un couple scolaire. EspacesTemps : Les Cahiers, Vol. 66-67, p. 55-64.

Ricoeur P., 2006. Devenir capable, être reconnu. Texte écrit pour la réception du Kluge Prize (Bibliothèque du Congrès), http://www.diplomatie.gouv.fr/fr/IMG/pdf/Revue_des_revues_200_ 1152AB.pdf

Sansot P, 1985. La France sensible. Paris, Champvallon.

Todd, E., 2015. Qui est Charlie ? Sociologie d'une crise religieuse. Paris, Éditions du Seuil.

\section{NOTES}

1. C'est toute cette démarche, de l'épreuve de la nécessité de l'enquête, au travail critique sur les résultats qu'elle apporte, confrontés à des connaissances établies par ailleurs, qui constitue alors le commun.

2. Bailly, 2011, p. 9.

3. Une exception notable est le rapport réalisé à la demande du Bureau des paysages du ministère de l'Écologie, du Développement durable et de l'Énergie, portant sur l'étude du corpus photographique réuni à l'occasion du concours Mon paysage au quotidien, La France vue par ses habitants. Ce concours destiné aux particuliers et aux scolaires a rassemblé 8000 contributions qui permettent d'apprécier l'évolution de la perception du territoire national. Les chercheurs 
observent la prédominance d'un paysage patrimonialisé ainsi que d'un paysage idéalisé d'où les marqueurs du quotidien sont absents (Bertho et al., 2014).

4. Les programmes ont été composés par cycles redéfinis dans une intention de travailler à la continuité des apprentissages entre école et collège. Ainsi, le cycle 3 comprend les deux dernières années de l'école primaire et la première année du collège; le cycle 4 correspondant aux trois autres années du collège.

5. Interviewé par François Jarraud pour le Café pédagogique le 09 février 2015, Michel Lussault précise : «Les enseignants se l'approprieront comme ils le souhaiteront et le transformeront en activités d'apprentissage ».

6. La critique de l'essai d'Emmanuel Todd par le géographe Thierry Joliveau est un modèle du genre et peut servir de référence en la matière (Joliveau, 2015).

7. L'écriture des programmes d'histoire fait moins de place que ceux de géographie aux élèves. Mentionnés quatre fois en histoire dans les « démarches et contenus d'enseignement », les élèves le sont dix-huit fois en géographie.

8. L'enseignement de la géographie conserve pour 2016 la distribution initiée en 2008 avec la notion d'habiter en classe de sixième ; l'inégal développement, les ressources et le changement global en classe de cinquième; les dimensions géographiques de la mondialisation contemporaine en classe de quatrième ; la France et l'Union européenne en classe de troisième.

9. Il faut cependant noter la surprenante disparition de la région comme thème d'étude. Deuxième thème de la première partie du programme de 2008, elle n'apparait plus en 2016 que comme une échelle possible dans une étude de cas d'aménagement local et/ou régional (deuxième partie du programme) et dans "l'examen d'une région transfrontalière » (troisième partie).

10. Le domaine $\mathrm{n}^{\circ} 3 \mathrm{du}$ socle, La formation de la personne et du citoyen, est présentée comme devant permettre « les choix et l'engagement éthique des personnes» (premier paragraphe).

11. Au sens où une norme intériorisée par les élèves interdit qu'il soit fait mention de ces espaces à forte valeur familiale et culturelle.

\section{ABSTRACTS}

The focus of the debates on the national narrative in history erased the political stakes in the class of geography in the secondary education. The public success of works which propose geographies of France shows nevertheless the interest for the geographical narrative. We analyze the contents of these works and we identify the stakes in the teaching of the French society in geography to the middle school. An ambivalent impression emerges from it. On one side, a professional ethics is possible and it would attempt to think of the disparities produced in and by the teaching of the geography. On the other hand, a school disciplinary reproduction is possible and will not try to think of these disparities.

En se focalisant sur le récit national, les débats sur les programmes d'histoire ont effacé les enjeux politiques du récit en classe de géographie. Or, l'actualité éditoriale montre le succès public d'essais de type récit géographique de pays. À partir d'une analyse de leur contenu, on identifie les enjeux de l'enseignement, au collège, de la géographie de la France. L'étude des programmes de 2016 laisse une impression ambivalente. D'un côté, peut s'esquisser une éthique professionnelle s'attachant à penser les inégalités produites dans et par l'enseignement de la 
géographie. De l'autre, persiste le risque d'une reproduction disciplinaire qui tend à les ignorer et les entretient.

\section{INDEX}

Mots-clés: Géographie scolaire, enseignement secondaire, géographie de la France, récit, éthique professionnelle

Keywords: School geography, secondary education, geography of France, narrative, professional ethics

\section{AUTHOR}

\section{JEAN-FRANÇOIS THÉMINES}

Jean-François Thémines, jean-francois.themines@unicaen.fr, est Enseignant-chercheur à l'Université de Caen Normandie et membre de ESO UMR 6590. Il a publié récemment :

- Thémines J.-F., Le Guern A-L, 2016. Initiation à la recherche, formation initiale et formation continuée : une analyse didactique du travail enseignant en géographie. In Ethier A.-M. et Mottet E. (dir.), Didactiques de l'histoire, de la géographie et de l'éducation à la citoyenneté. Bruxelles, de Boeck, p. $157-172$.

- Thémines J.-F., Caro P., 2015. Normandie sensible d'Armand Frémont. Tableaux d'une égogéographie. Géographie et cultures, nº 89-90, L'Harmattan, p. 77-91.

- Thémines J.-F., 2015. Entre discipline et métier : des professeurs d'histoire-géographie face à la professionnalisation. In Bodergat J.-Y. et Buznic-Bourgeacq P., Des professionnalités sous tensions. Bruxelles, De Boeck, Coll. Perspectives en éducation et en formation, p. 169-187. 